\title{
Intra-day Electro-Thermal Model Predictive Control for Polygeneration Systems in Microgrids
}

\author{
Ramanunni P Menon ${ }^{\mathrm{a}, \mathrm{b}, 1}$, François Maréchal ${ }^{\mathrm{a}}$, Mario Paolone ${ }^{\mathrm{b}}$ \\ ${ }^{a}$ EPFL SCI-STI-FM,ME A2 434, Station 9, CH-1015 Lausanne \\ ${ }^{b}$ Distributed Electrical Systems Laboratory (DESL), EPFL-STI-IEL-DESL,ELL 116, Station \\ 11, CH-1015 Lausanne
}

\begin{abstract}
This paper is framed within the context of intra-day optimal control of polygeneration systems and storage connected to microgrids. In particular, the paper proposes an optimal control strategy that accounts for both electrical and thermal processes taking place in multi-building energy networks. To this end, the proposed optimal control strategy, based on the use of a Model Predictive Control, has been developed with the aim of providing optimal resource set-points for the coming 24 hours. The proposed approach takes into account: day-ahead and spot prices of electricity, resource prices in terms of fuel cost and shut-down, start-up costs as well as the presence of electrical and thermal storage.

The ability of this thermo-electric optimal control strategy is then demonstrated through simulations considering three case studies, namely: (a)fixed electricity price, (b)electricity spot-price and (c)different seasonal cases. The paper also studies the shaving of peaks through the use of optimally sized units in a system with the proposed method.
\end{abstract}

Keywords: Polygeneration systems, Model Predictive Control, Microgrid, CIGRE, Energy management

Email address: ramanunni.menon@epfl.ch (Ramanunni P Menon)

$U R L$ : ipese.epfl.ch (Ramanunni P Menon)

${ }^{1}$ Corresponding Author

Preprint submitted to Energy

April 15, 2016 


\section{Nomenclature}

$\Delta T$ Temperature difference

$\dot{E l}$ Electric Active Power

$\dot{m} \quad$ Mass flow(fuel or streams)

$\dot{Q} \quad$ Thermal Power

$\eta_{t h}$ Thermal efficiency for CHP units

$\mathcal{N}$ Number of buses of the electrical grid

$\mathcal{S}$ Number of slack buses of the electrical grid

$\tau_{y, i}$ Annualised interest

$c_{r}$ Cost

CHP Combined Heat and Power unit

$\mathrm{CO}_{2}$ Yearly Carbon dioxide emissions

COP Coefficient of Performance of Heat Pumps

E Voltage

EIB European Installation Bus

$H P$ Heat Pump

I Investment Cost

$\operatorname{Im}[S]$ Reactive Power

INVC Yearly Investment Cost

$J \quad$ Jacobian matrix used in Newton-Raphson method

MFH Multi-Family House

MOO Multi-Objective Optimisation 
MPC Model Predictive Control

$n \quad$ Number of Nodes

$O P C$ Yearly Operating Cost

$R E[S]$ Active Power

$s \quad$ Number of storage tanks

SFH Single Family House

SIA Schweizischer ingenieur -und architektenverein(Swiss Society of Engineers and Architect)

SOC State of Charge

$t \quad$ Time

$T_{\min }$ Minimum switch on time

$T C$ Total Cost

$u \quad$ Number of units/devices in system

$V \quad$ Volume of storage

$V 2 G$ Vehicle-to-Grid Implementation

VPP Virtual Power Plant

$y_{x}$ Integer variables for $(\mathrm{x}=$ start-up)start-up and $(\mathrm{x}=\mathrm{on})$ switch on

$C_{s, n}$ Sizes of the storage devices in the system

$C_{u, n}$ Sizes of the units in the system 


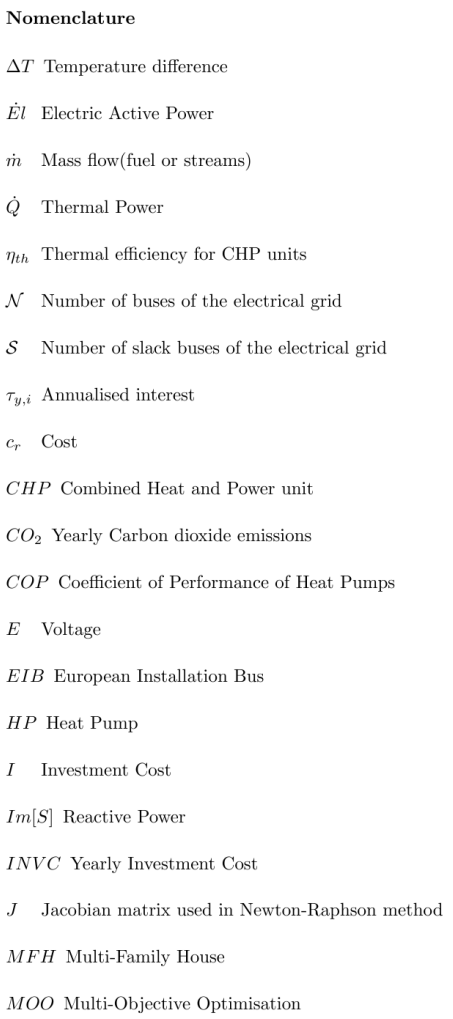

(a) Nomenclature, Part 1

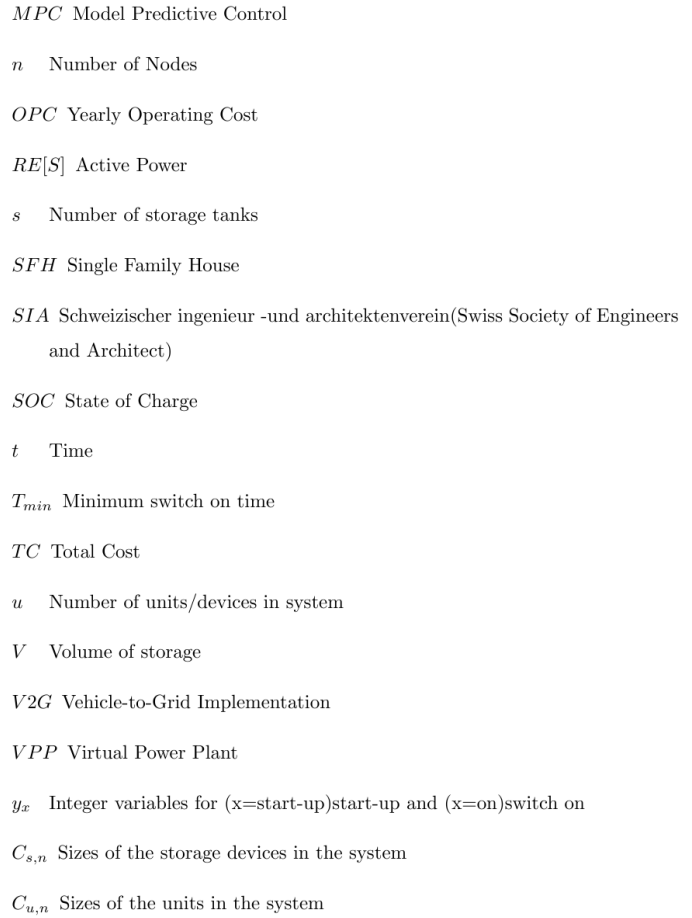

(b) Nomenclature, Part 2

Figure 1: Nomenclature used for the equations for the following sections. 


\section{Introduction}

The evolution of the electrical and thermal demands, together with the need of highly efficient systems, is changing the paradigm of electrical distribution grids towards systems capable of importing and exporting power/energy. Within

5 justifies the investigation of specific control strategies that account both these systems in order to maximize the overall efficiency by maintaining the electrical grids within their safe operation conditions.

Modern grids make use of a mix of deterministically dispatchable units like: newable resources,must satisfy the consumer's demand. These devices, along with the use of thermal and electric storage systems, are also used to maximize the entire system efficiency. Microgrids represent a promising integration platform for all the above-mentioned elements. They can, indeed, use these ing the entire system (e.g. Lasseter et al.[1]). In a scenario without Demand Side Management (DSM), the stochasticity in the user demand requires the use of a robust control strategy to achieve, for instance the maximum microgrid efficiency, [2]. MPC (Model Predictive Control) is one of the most adopted Kriett et al. and Prodan et al [3], [4] and [5]. For an MPC strategy, optimal sizing of the components in the system is vital. However, such a task cannot be decoupled from the definition of the optimal control strategy. Within this context, this work aims at proposing a methodology for the optimal control of mal predictive control is handling the thermal and electrical power flows. Most studies([6], [7],[8],[9],[10] and [11]) as has been shown in great detail in Section 2 , conducted till now, either focus on the thermal flows or the electrical flows owing to the differing inertia and the resulting dynamics. The paper is putting

forward an MPC strategy that is able to not only integrate both the flows, but 
also maintain the quality of said electrical and thermal flows in the grid within standards demanded by industry and improve the total system efficiency, while satisfying the comforts of the end-users. For the purposes of proper assessment, this model predictive control strategy has been tested on an IEEE microgrid test benchmark([12]). The paper also shows how to couple the proposed MPC strategy with a sizing process of the cogeneration, heat pumps and thermal storage devices. The validation of the proposed MPC is carried out through the use of a specific microgrid under development at the author's laboratory. The paper is structured as follows: Section 2 clarifies the need for an integrated approach of control for a microgrid using references as a yardstick for the control strategy demonstrated in this paper and further details the scientific originality and novelty of the paper, Section 3 gives a concise explanation of the optimal sizing problem, Section 4 describes the methodology employed and the equations associated with the methodology, Section 5 shows the system by describing its different elements, Section 6 explains the different simulations that have been performed and the adopted conditions. The final two sections explore and analyse the results and provide conclusions.

\section{Need for an integrated thermal-electrical control in Active Distri- bution Networks}

The promotion of renewable energy resources (RERs) and, in general, distributed generation (DG) has resulted in an increasing attention by all stakeholders on electrical distribution networks (DNs). The number of connections of energy-resources to DNs is rapidly increasing in a way such that, in several countries, operational constraints are already attained. In this respect, the progressive penetration of DG into electrical medium and low voltage networks will cause, in principle, an important re-engineering of this part of the electrical infrastructure. One of the main obstacles preventing maximum penetration of traditional and renewable distributed energy resources (DERs) into DNs is 
represented by the lack of optimal control strategies for DERs by distribution networks operators (DNOs).

An important element that must be considered is the need of control of thermal loads since they represent, in general, the major elements of demand for both residential and industrial customers. The paper aims at demonstrating that a suitably-defined optimal control strategy that takes into account both of the above mentioned aspects, can achieve the maximisation of the global system goals like end-user comforts, energy savings, grid efficiency and economic savings.

The thermal/electrical grids have CHP units and storage systems which have the ability to interact with the system as and when needed and thus, can be used to provide support to the system needs. But, at the same time, without proper control, the stochastic sources and CHP units supplying into the system have the potential to create non-optimal or critical operation conditions which can/may be able to take down parts of the grid or cause massive losses of electrical power.

At the same time, users receiving the power, both thermal and electric, need to be satisfied and enjoy the comforts from these devices. This necessitates the need for an effective control strategy for the control of both the thermal and electrical flows, such that the electrical and thermal flows satisfy the comfort so defined for the user, while providing stability and improving efficiency.

Several authors have proposed various control strategies in this context. However, most of the papers that deal with this topic have shortcomings or have a tendency to overlook one of the many aspects that all need to simultaneously included for a full and complete control strategy. Most of the papers continue to deal with the problem as either an electric or a thermal one, which leaves much to be desired. For example, Katiraei et al. [6] and Barklund et al. [7] outline the large number of microgrid management techniques, mainly from the power management side involving voltage and frequency controls, but this does not involve forecasting and management with a larger picture in purview. 90 Parisio et al.,[8],Kriett et al.[5], and Collazos et al. [3] all provide similar ap- 
proaches with subtle differences for an end-user in a grid. Collazos et al. [3] propose a MPC strategy that studies the problem from a thermal perspective but, omits electrical storage and power flow calculations, Kriett et al. [5] use the electrical devices as well, but fail to include part-load characteristics of the CHP units and heat pumps used in the systems and uses simplified electrical flows while sacrificing electrical and thermal buffers. Parisio et al.[8], use similar approaches to both Collazos et al. and Kriett et al., but use quadratic cost functions which provide a much more detailed and accurate representation of the objective function, but runs the risk of a high computation time and local minimas depending on full simulations. Stluka et al.[13] show another method of economic allocation of energy by the units to the consumer, but fail to account for the thermal energy supply from the CHP units and do not consider the use of short-term storage of electrical energy through the use of heat pumps and thermal storage devices. This along with mass storage is a powerful method for the microgrid to satisfy the demand and yet, minimise the operating and total costs in the long run. Hooshmand et al.,[9] provide a stochastic MPC model for the microgrid, using stochastic sources, but does not take into account the thermal flows. Garcia et al.[10] and [11] show a method for using the day-ahead electricity market prices and make use of stochastic renewable energy resources fitted with storage devices using Model Predictive Control. All these papers show one part of the problem. In this respect, this paper attempts to show a Model Predictive Control strategy which accounts for the electrical and thermal flows, yet also, includes the different storage devices, the varying electrical prices and the electrical flow calculations and cost functions on a grid framework which is based on a LV(Low Voltage)-microgrid benchmark.

This paper contains an evolution of the work presented in [3]. The original and new contributions of this paper are:

- The addition of heat pumps and electrical storage devices to the problem

- Part-load equations for the differing heat and electricity production of the CHP units and the heat pumps 
- Inclusion of the electric power flow calculus

- Variables to view and control the electrical and thermal storage potentials and manipulate the supply or demand of a unit or end-user.

- Use of the CIGRE microgrid test benchmark for the performance assessment of the proposed control strategy.

The units used for the control system have been optimally sized using a methodology which integrates the multi-objective optimization using a genetic algorithm developed by [14] with the proposed control strategy. This optimal sizing methodology has been demonstrated in [15](Menon et al.), where the effect of different constraints on sizing have been shown. The following section elucidates the methodology of the strategy employed for deciding the set-points and satisfying the demands of the loads. In summary, the novelty of this paper is that it provides a more complete analysis of the electrical and thermal units and devices used by end-users and highlights the interconnectedness between these devices and use these factors in an MPC strategy that is able to create daily strategies for the power consumptions, both electric and thermal, for a low voltage network of consumers while satisfying the comforts of these consumers and minimising their operating costs and total costs. The system being studied and referenced through the rest of the paper is based on the generic topology shown in Figure 2. As can be seen, it is composed of different levels, with both thermal and electrical power flows through the different levels. The smallest unit for calculation is the node at which various devices can connect. The node is a fluid connection, so it could be connected to one energy source or unit or to a multitude of devices and end users.

\section{Sizing}

Fig.2 shows the schematic of the microgrid that is the system studied. The various units connect to the microgrid at nodes which act as the basic interacting unit of the microgrid. It interacts with the rest of the system and depending on 


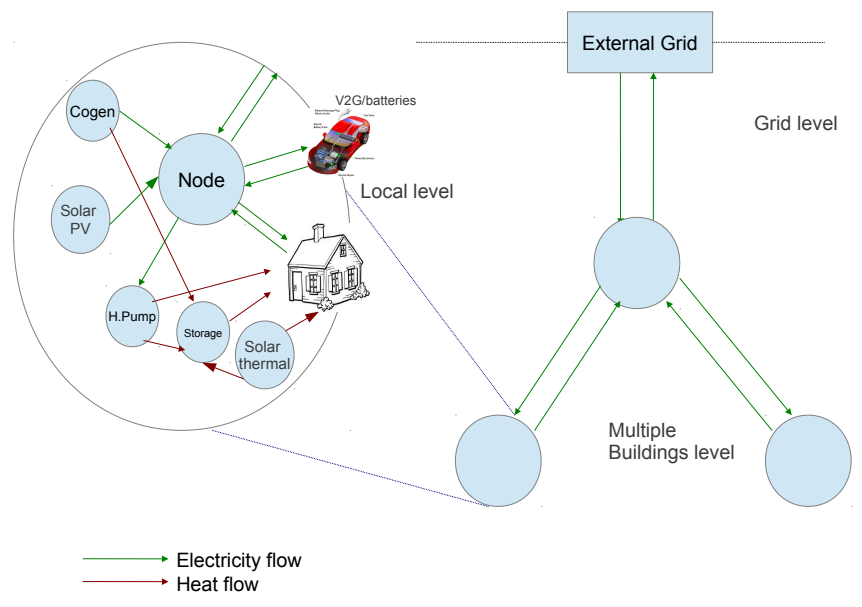

Figure 2: Schematic of the microgrid

the position of the node in the microgrid, the flows coming in and going out can vary. In the figure, the node is interacting with the house and the cogeneration unit with both electrical and thermal flows. For the efficient functioning of the system as a whole, each of these units interacting with the node needs to be sized.

As mentioned in the previous section, the optimal sizing of the components has been done by solving a Multi-Objective optimisation(MOO) using an algorithm developed by [14]. This implementation has been performed and published in the work, [15]. Using equations that describe the system and typical and extreme days of the year which provides data about the demands of the system, the Multi-Objective optimisation uses a genetic algorithm to simultaneously minimise the operating cost of the system/node, the total costs of the system and the carbon emissions of the system. The decision variables of the optimisation problem include the sizes of the resources/devices in the nodes. This is then used to plot a Pareto curve. The system that best fits the case studied is picked out of the pareto curve and the corresponding sizes of the units are used in the Model Predictive Control. 


\section{Methodology}

The model predictive control involves solving a MILP(Mixed Integer Linear Programming) problem which aims at minimising an objective function which is, usually, the operating costs or alternatively, the carbon emissions of the system components of the system and the emissions.

As shown in Fig.3, the problem has been divided into two sub-problems. The need for this separation is due to the presence of both thermal and electrical devices in the microgrid and the buildings. As the thermal and electrical processes day.

The first sub-problem involves solving a global MILP to create supply and demand profiles and setpoints for each hour in the next 24 hours such that the devices and the grid optimally satisfy the electrical and thermal demand of the loads/users. The input for this sub-problem consists of all temperature inputs, internal and external for the starting time $t_{0}$, the state-of-charge of the storage devices, the demand from the loads, for time $t_{0}$ and also, the states of the CHP units and the heat pumps and any other unit in the system.

The equations that are used to represent the thermo-electric processes of the 


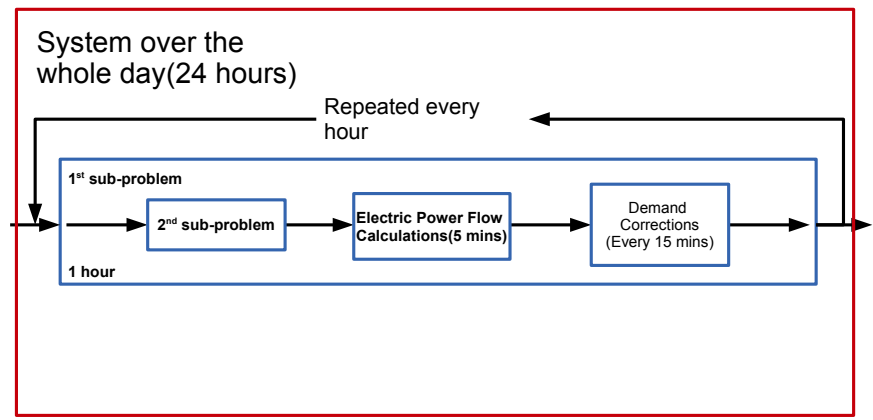

Figure 3: Schematic of the problem

cogeneration units, storage devices ${ }^{2}$ and heat pumps are given below:

$$
\begin{aligned}
& \dot{Q}_{c g}(t)=y_{c g}(t) \cdot \dot{Q}_{c g}^{\max } \\
& \dot{E} l_{c g}(t)=y_{c g_{o n}}(t) \cdot \dot{\boldsymbol{E}}_{\boldsymbol{c g}}^{\text {min }}+y_{c g_{s t a r t-u p}} \cdot \eta_{\boldsymbol{c g}, \boldsymbol{e l}}^{\text {start-up }} \cdot \dot{\boldsymbol{H}}_{\boldsymbol{c g}}^{\max } \Delta t \\
& +y_{c g_{p w}}(t)\left[\boldsymbol{m}_{\mathbf{1}}^{e l}\left(\dot{Q}_{c g}(t)-\dot{\boldsymbol{Q}}_{c \boldsymbol{g}}^{\text {min }}\right)+\dot{\boldsymbol{E}}_{\boldsymbol{c g}}^{\boldsymbol{m i n}}\right]+ \\
& \left(1-y_{c g_{p w}}(t)\right)\left[\boldsymbol{m}_{\mathbf{2}}^{e l}\left(\dot{Q}_{c g}(t)-\dot{Q}_{c g, p w}\right)+\boldsymbol{m}_{\mathbf{1}}^{e l}\left(\dot{Q}_{c g, p w}-\dot{\boldsymbol{Q}}_{c g}^{m i n}\right)\right] \\
& \dot{H}_{c g}(t)=y_{c g_{o n}}(t) \cdot \dot{\boldsymbol{H}}_{\boldsymbol{c g}}^{\min }+y_{c g_{s t a r t-u p}} \cdot \dot{\boldsymbol{H}}_{\boldsymbol{c g}}^{\max } \Delta t \\
& +y_{c g_{p w}}(t)\left[\boldsymbol{m}_{\mathbf{1}}^{g a s}\left(\dot{Q}_{c g}(t)-\dot{\boldsymbol{Q}}_{c \boldsymbol{g}}^{\text {min }}\right)+\dot{\boldsymbol{H}}_{c \boldsymbol{g}}^{\text {min }}\right]+ \\
& \left(1-y_{c g_{p w}}(t)\right)\left[\boldsymbol{m}_{\mathbf{2}}^{\text {gas }}\left(\dot{H}_{c g}(t)-\dot{Q}_{c g, p w}\right)+\boldsymbol{m}_{\mathbf{1}}^{\text {gas }}\left(\dot{Q}_{c g, p w}-\dot{\boldsymbol{Q}}_{c \boldsymbol{g}}^{\text {min }}\right)\right] \\
& y_{c g_{o n}}(t) \in\{0,1\} \\
& y_{c g_{p w}}(t) \in\{0,1\}
\end{aligned}
$$

\footnotetext{
${ }^{2}$ all listed below for conciseness as they are similar to the equations cited in [3]
} 


$$
\begin{gathered}
\dot{E} l=\frac{\dot{Q}}{C O P} \\
C O P=\frac{\eta_{C O P}}{1-\frac{\Delta T^{\text {cold } l m}}{\Delta T^{h o t} l m}}
\end{gathered}
$$

Equation 1 provides the link using the binary integer variable between the thermal and/or electrical output and the switching on/off of the device equations. Equations 2 and 3 model the change of power output depending on the switching on and off of the devices with time. As heat pumps and cogeneration units are characterised by output power equality constraints that are non-convex, it has been decided to adopt piecewise function approximation to represent changes in thermal and electrical outputs with time. This provides us with a better correlation to the resulting heat/electricity provided to the end-user. 4 and 5 shows us the binary nature of the above-mentioned linear-piecewise functions and switch-on and off variables.

Equation 6 represents the electrical power required for the thermal power output as a function of the COP and the thermal power output by the unit and equation 7 expresses the COP as a function of the efficiencies and the mean log tempereature difference between the hot and cold sources as per [16] Girardin et al..

For the CHP units, the slopes for piece-wise functions representing the partload conditions are represented using $m_{1}$ and $m_{2}$. The thermal storage devices are water tanks integrated between the conversion system and the heat distribution system of the house. A three valve system is considered. It is assumed that the power delivered by the unit and considered by the conversion unit can be either realised by a level of operation (part load) or by a reduced operating time at nominal power during the period. The storage tank models includes a heat loss model that is a function of the temperature in the tank. The electrical storage devices have a (State of Charge) SOC, which varies from 0 to 1 , which is used as a multiplying factor to the total capacity of the storage devices and the charging and discharging curves. 
The equations formulating the MPC are:

$$
\begin{aligned}
& \forall u \in \text { Units, } \forall n \in \text { Nodes } \Rightarrow C_{u, n} \\
& \forall s \in \text { Storage, } \forall n \in \text { Nodes } \Rightarrow C_{s, n} \\
& \left.I N V C=\sum_{n=1}^{\text {NodesUnits }} \sum_{u=1}^{1} \frac{1}{\tau_{y, i}}\left(\text { Sizes }_{u, n}\right)+I\left(V_{s, n}\right)\right) \\
& \forall s \in \text { Storage } ; \forall n \in \text { Nodes } ; \forall t \in \text { Time } \Rightarrow \dot{m}_{u, n}(t), V_{s, n}(t) \\
& O P C=\int_{t=1}^{\text {Time }}\left(\sum_{u=1}^{\text {Units }}\left(\left(c_{r}^{+}(t) \dot{(m}\right)_{r(u), n}(t)\right)+\left(c_{r}^{+}(t) \dot{E}^{+}(t)\right)\right) d t \\
& T C=O P C+I N V C
\end{aligned}
$$

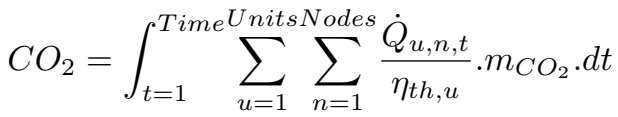

$$
\begin{aligned}
& y_{x} \in\{0,1\} \text { where } x=\text { on/start }- \text { up } \\
& y_{\text {start-up }}(t) \geq 0 \\
& y_{\text {start-up }}(t)=y_{\text {on }}(t+\Delta t)-y_{\text {on }}(t) \\
& \sum_{r=t+\Delta t}^{t+T_{\text {min }} o n} y_{o n}(r) \Delta t \geq y_{\text {start-up }}(t) \cdot T_{\text {min-on }}
\end{aligned}
$$

The global problem is solved in the form of a MILP problem which involves the use of the minimisation of the global operating costs such that the systems and units satisfy the user demands. The solution of the problem provides the integer variables for the thermal and electrical devices for the coming 24 hours. 
Equations 8, 9, 10 and $11 \& 12$ shows us the global problem in concise terms. 8 and 9 shows us the cost of every unit and system in each of the nodes. Equations 10 and 12 show the associated investment and operating cost for the entire system at any moment in time. The operating costs are represented by the summation of all the costs of buying and selling resources, electric and thermal energy and the associated costs of not satisfying the demands of the end-user. This associated cost accounts for the comfort of the user in the problem and, for each solution that does not satisfy, there is an associated penalty. The objective function maybe also variable depending upon the requirement of the user. The total cost is calculated by combining the investment cost and operating cost. The carbon emissions for each unit and for each time is calculated and summed using 14. With respect to the adopted case studies, the objective function chosen is represented by the operating cost only. The integer variables for start-up and shutdown of units in the microgrid are listed in 15-18. These equations also show the limits on the number of start-ups possible within a period of time. This is inserted in the problem to prevent too many start-ups and shut-downs within a small period of time. This is to avoid, sub-optimal performance of the units.

$$
\begin{gathered}
\sum_{t=1}^{\text {Time }} \sum_{n=1}^{\text {Nodes }} \sum_{h=1}^{H} \dot{Q}_{h, n, t}^{+} . d t=\sum_{t=1}^{\text {Time }} \sum_{n=1}^{\text {Nodes }} \sum_{h=1}^{H} \dot{Q}_{u, n, t}^{-} . d t- \\
\sum_{t=1}^{\text {Time }} \dot{Q}_{\text {loss }, t} . d t \\
\sum_{n=1}^{\text {Nodes }} \sum_{h=1}^{H} \dot{E} l_{h, n}^{+}(t)+\dot{E} l_{h p}^{+}(t)=\sum_{u=1}^{\text {Units }} \sum_{n=1}^{\text {Nodes }} \dot{E} l_{u, n}^{-}(t)+ \\
\dot{E} l_{\text {grid }}^{+}(t)+\dot{E} l_{\text {grid }}^{-}(t) \\
\min _{Q_{n}, y_{n}}\left[O P C+I N V C, C O_{2}\right] \text { s.t.min } Q_{Q_{n, t}, y_{n, t}} T C \\
\min _{Q_{n}, y_{n}}\left[O P C\left(y_{n}\right)\right]
\end{gathered}
$$




$$
\begin{array}{r}
\forall t, \psi^{\prime}\left((1-S O C) \cdot \dot{E} l_{s t}+C_{t}\right) \leq \dot{E} l_{\text {give }}(t) \leq \\
\chi^{\prime}\left(\left(1-y_{c g}\right) \dot{E} l_{c g}+(1-S O C) \dot{E} l_{s t}+C_{t}\right) \\
\forall t, \psi\left(y_{c g} \cdot \dot{E} l_{c g}+S O C \cdot \dot{E} l_{s t}\right) \leq \dot{E} l_{\text {accept }}(t) \leq \\
\chi\left(\dot{E} l_{c g}+S O C \cdot \dot{E} l_{s t}\right)
\end{array}
$$

Equations 19 to 24 model the flow of energy in the systems. As the flows of electrical and thermal power flows are directly related to the units in the system and the integer variables themselves, these flows are such that the summation completely balances the requirements of the user and the power supplied by the devices while accounting for the losses in the transfers.

The second sub-problem involves electrical power-flow calculations to correct the hourly predictions in order to maintain the electrical grid quality of supply. In what follows, the quality of supply is limited to the case of voltage quality since, in distribution networks, this is the most important quantity to be controlled. The thermal and electrical demand of the initial time is used as the starting point, and once the first hour prediction is available, the power flow calculations for the network is performed every 5 minutes. The heat pumps and the CHP units are treated as loads and sources respectively. Every 15 minutes, the thermal and the electrical demand is corrected based on the calculations with reference to the hourly predictions.

The electrical power flow calculations are performed using Newton Raphson method for an electrical network of known topology and admittance matrix. The changes in Heat Pump and CHP setpoints are computed by solving a convexified optimisation problem formulated using linearised dependences between voltage variations and nodal power injections. In particular, these sensitivities are calculated in correspondence of the interface with the external grid and in the nodes where the consumers and devices, are connected. The sensitivity coefficients are calculated based on the method proposed in [17]. The equations 25 to 30 illustrate the sensitivity coefficients and how they are calculated. The grid 
is treated as an active component that dictates how much supply and demand it requires over the period of time. The updated values are then notified to the higher problem and the values are then updated with a further optimisation.

$$
\begin{aligned}
& S_{-}=E_{-} \sum_{j \in \mathcal{S} \cup \mathcal{N}} \bar{Y}_{i} j \bar{E}_{j}, i \in \mathcal{N} \\
& \forall \text { bus } i,\left|\Delta V_{i}\right|=K_{i R e[S]} \Delta R e[S]+K_{i \operatorname{Im}[S]} \Delta \operatorname{Im}[S]+K_{i n} \Delta n \\
& \frac{\partial\left|\bar{E}_{i}\right|}{\partial(\operatorname{Re}[S])_{l}}=0, \forall i \in \mathcal{S} \\
& \frac{\partial\left|\bar{E}_{i}\right|}{\partial(\operatorname{Re}[S])_{l}}=\frac{1}{\left|\bar{E}_{i}\right|} \operatorname{Re}\left(E_{-} \frac{\partial \bar{E}_{i}}{\partial(\operatorname{Re}[S])_{l}}\right), \forall l \in \mathcal{N} \\
& \text { L.b. } \leq p . f .(\cos \phi) \leq U . b . \\
& \min _{R e[S], \operatorname{Im}[S]} \sum_{j=1}^{\mathcal{N}_{\mathcal{U}}} \alpha^{2}\left[\operatorname{Re}[S]_{j}-\operatorname{Re}[S]\right]^{2}+\sum_{i=1}^{\mathcal{N}} \gamma^{2}\left[E_{i}-\bar{E}\right]^{2}
\end{aligned}
$$

At the end of the each hour, the power flow calculations and the adjustments over the 15-minute intervals are are accounted and the actual power consumption is used as the input for the next hour and the same process is followed for each hour.

\section{Case Description}

The system uses a set of 6 houses and 2 apartments within one microgrid which has a CHP unit in 3 of the buildings, 3 have heat pumps attached to them, and the other 2 houses have both heat pumps and CHP units attached. Table 1 clearly mentions the type of CHP unit and heat pump that is attached to each house. All of the houses have thermal storage. The houses and apartments that have been taken into account are based on the SIA standards(SIASFH, SIA 380, 
SIAMFH etc.)[18]. The thermal storages only satisfy the thermal requirements of the buildings.

The houses with CHP units also have electrical storage systems. The electrical network uses a benchmark feeder for LV networks proposed by the CIGRE working group[12]. The network is shown in Figure 4. Each of the nodes and the node element values are given in the figure.

Table 1: System components

\begin{tabular}{|c|c|c|c|}
\hline & CHP Unit & Heat Pump & Storage \\
\hline House 1 & Stirling Engine & Air-Water HP & Electric and Thermal \\
\hline House 2 & Diesel Engine & Geothermal HP & Electric and Thermal \\
\hline House 3 & Stirling Engine & - & Electric and Thermal \\
\hline House 4 & PEM Fuel Cell & - & Electric and Thermal \\
\hline House 5 & - & Groundwater HP & Thermal Storage \\
\hline House 6 & - & Air-Water HP & Thermal Storage \\
\hline Apartment 1 & PEM Fuel Cell & - & Electric and Thermal \\
\hline Apartment 2 & - & Geothermal HP & Thermal \\
\hline
\end{tabular}

\section{Simulation, Results and discussion}

The optimally sized units and the buildings are input as parameters of the system and different simulations were performed using the MPC. The major aspects that have been studied are the differences in the strategies depending on the different seasons of the year and the different strategies that are implemented when the electricity and the natural gas prices are varied. The effect of the electricity price is studied in this paper through the use of two different set of simulations. For the first price simulation, fixed prices are considered and for the second one, variable prices are considered.

Our goal is to study how the system will behave when the electricity prices 


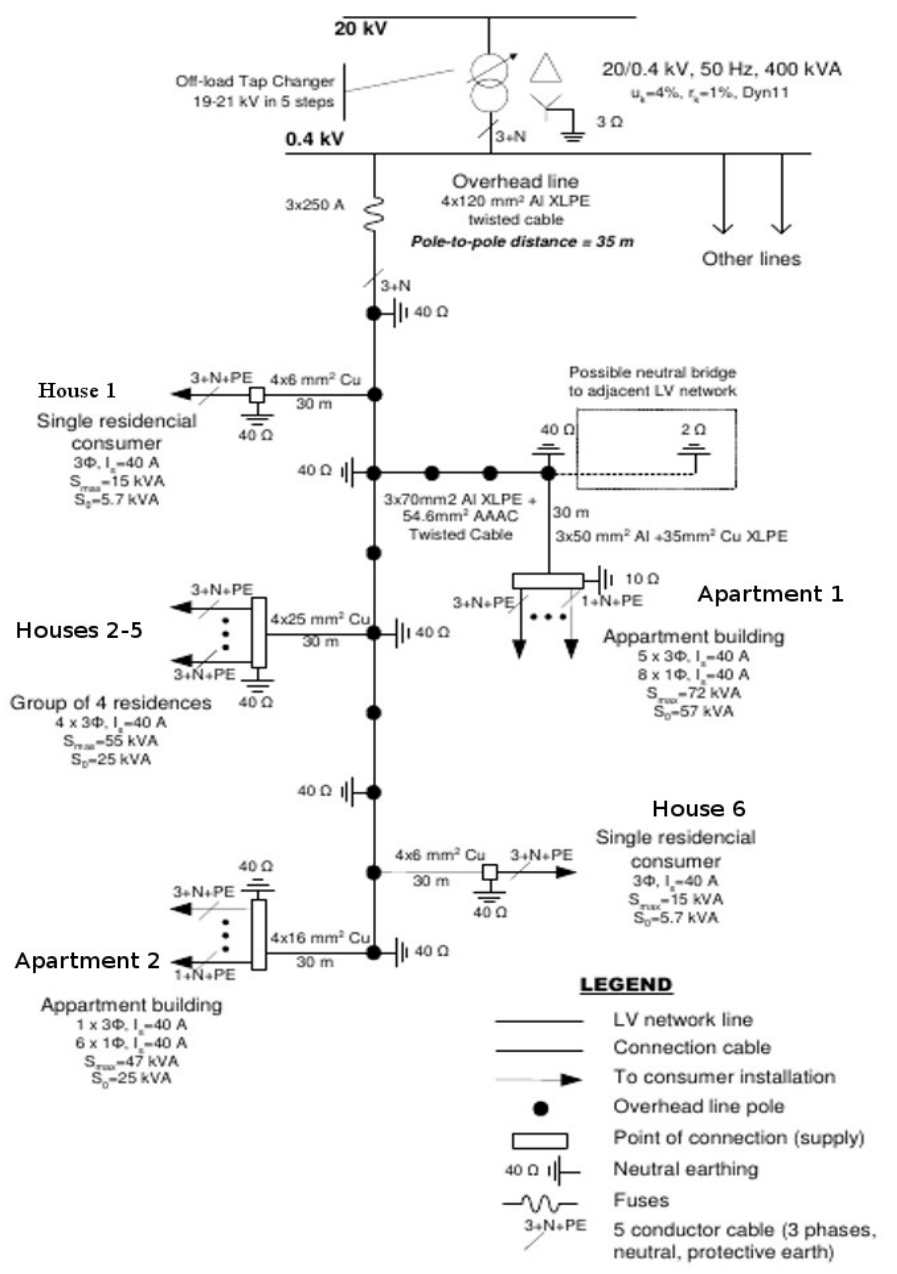

Figure 4: Structure of electrical network(Adapted from [12])

price profile for the system. We will then compare the energy consumption and the study the behaviour of the network when alternative electricity price profiles will be presented.

\subsection{Fixed Prices}

This simulation uses the existing scheme of electricity prices levied on the consumers and the system uses both electrical and thermal storage devices. All 
Table 2: Pricing schemes

\begin{tabular}{|c|c|c|}
\hline & Buying Price & Selling Price \\
\hline Fixed Price Scheme & $\begin{array}{c}€ 0.14 / \mathrm{kWh} \text { for } 6 \mathrm{AM} \text { to } 10 \mathrm{PM}, \\
€ 0.08 / \mathrm{kWh} \text { for 10PM-6AM }\end{array}$ & $€ 0.07 / \mathrm{kWh}$ \\
\hline Variable Price Scheme & Spot buying prices & Spot selling prices \\
\hline
\end{tabular}

other variables are the unchanged for both price simulations. The simulation is performed for 2 different days, one in summer and one in winter to show the impact that price on the strategies.

The first simulations that were studied was the case of a single family house in the microgrid which has a cogeneration unit, a heat pump and storage devices(electrical/thermal). The electricity purchase and sale prices are fixed and are listed in Fig.5. This is similar to the price levied from the end-users in a LV-grid today and the strategy created for summer and for winter reflects the influence of the pricing of electricity prices. The initial predictions created for the day can be seen in Fig.12 for a given day in summer and Fig.15 for a given day in winter. Fig.13 and Fig.16 show the final consumptions and productions by each unit for house 1 after the predictions are altered by the electical subproblem to maintain quality of supply. The fact that the gas prices are lower than the purchase or sale prices of the electricity influences the strategy to a large extent, making the use of the cogeneration unit, which uses natural gas to produce electricity and heat, a more attractive option . During summer, the heat pump use is minimised to the times when electricity price is minimum. During winter, the use and impact of heat pump can be clearly seen. This results in the reduced use of the cogeneration unit for heat and subsequently leads to the lowered electricity production. The corresponding heat production and storage profiles for 24 hours for the different price profiles can be seen in figures 10 and 11. Figure 10 shows the heat storage profiles for summer for House 2 and figure 10 shows the profile for winter cases.

The figures so far have all been showing the result from the perspective 
of House 2 and the ability of the MPC to successfully create strategies for electrical and thermal resources for a given day in summer and winter. In this respect, figures 7 and 8 show the MPC strategies created for cogeneration units and heat pumps in other houses of the microgrid for the same day in summer with fixed prices, such that these devices aid the optimal satisfaction of the load demand profiles. These figures provide a side-by-side comparison of the different production profiles among the units in the system ( The type of devices are given in table 1). These two figures show the general similarities in strategies for similar sized houses for a given day in different parts of the grid within close proximity. This is owing to the general similarity of consumption patterns in different sectors of the grid. At the same time, it also brings out the uniqueness of each house and the differences in the consumption profiles due to the tiny differences in the temperatures and the consumption of the users in the house. These results shows the ability of the MPC to provide unique strategies depending on the location, the type of building and variations in prices and temperatures and user behaviour. The size of each unit in the various buildings computed by the sizing problem and used for the MPC, are shown in Figure 6. As can be seen, the cogeneration and heat pump sizes are shown in $k W_{e}$ and the capacities of thermal storage is shown in $m^{3}$ and 325 the electrical storage in $k W h_{e}$. Figure 9 further delves into and provides the differences in the sizes and the associated total costs (investment plus operating costs for a year $\left(10^{4} \mathrm{CHF} /\right.$ year $\left.)\right)$ for House 2 as a result of the use of the MPC strategy in the microgrid. These sizes and costs show that spot prices require larger investment costs due to larger unit sizes owing to a bigger role played by the CHP units. Similarly, the size of the CHP units with PV decreases for cogeneration units while increasing slightly for heat pump devices. Although, the presence and absence of PV has been analysed for sizing and total cost here, the effect of PV on a house with MPC strategy will be explored in a subsequent study.

Fig.14 shows the 24-hour voltage profile for the network for the day in summer with a direct comparison with the profile without voltage control. The day 


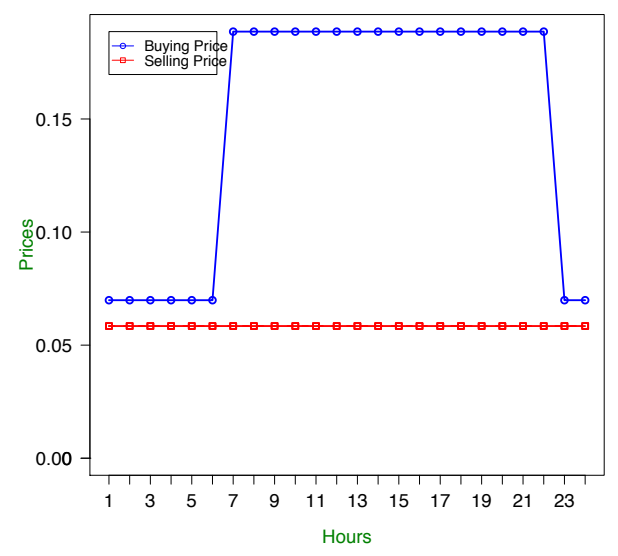

Figure 5: Graph of fixed pricing for consumers in present-day grid

in question is within the range of $+/-5 \%$ of the voltage deviations, but when the electrical interactions are calculated and entered into the MPC strategy, the voltage profile is clearly better and the resulting strateg is also better.

\subsection{Variable Prices}

These set of simulations uses the spot prices from the day-ahead market instead of the existing prices levied on the consumers and the system uses both electrical and thermal storage devices. Similar to the previous simulation, two simulations are performed, one for summer and one for winter.

The second set of simulations conducted where for the same day of the year so as to make a direct comparison with previous sub-section. The varying prices used are from the spot markets of Switzerland for the year 2012. The prices can be seen listed in the 17. The impact of using the spot prices is immediately seen in the corresponding strategies for summer and winter. The fact that electricity prices are lower, makes using the heat pump during the cheaper periods more lucrative during winter and maximising the use of the cogeneration unit when the prices are lower for the both winter and summer. Due to the fact, that there is a slight shift in the price towards the end of the day, the use of cogeneration, 


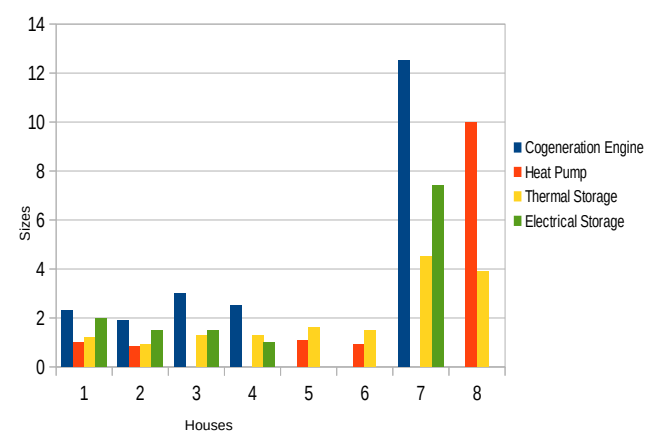

Figure 6: The sizes of each unit as per table 1 in the microgrid, the cogeneration units in $k W_{e}$, the heat pump in $k W_{e}$, the thermal storage in $m^{3}$ and the electricals storage in $k W h_{e}$

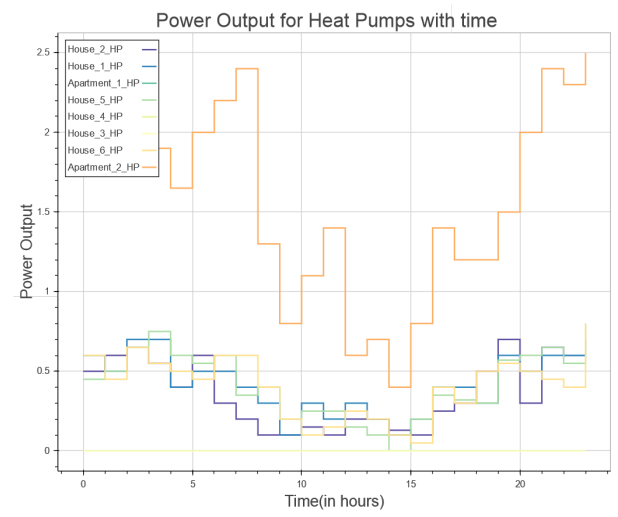

Figure 7: Heat pump electric power distribution for different houses in the microgrid

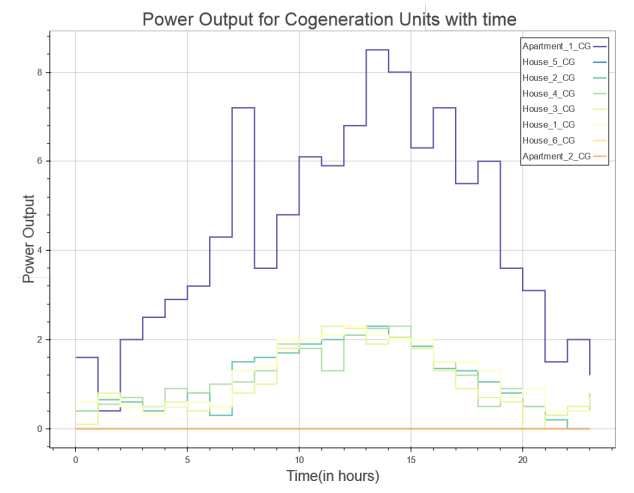

Figure 8: Cogeneration unit power distribution for different houses in the microgrid 


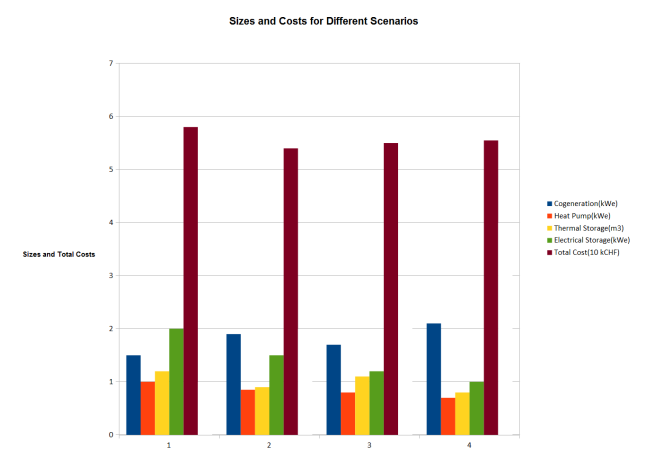

Figure 9: Costs and sizes for House 2 in 1. With PV; 2. No PV; 3. Spot prices; 4. Fixed prices

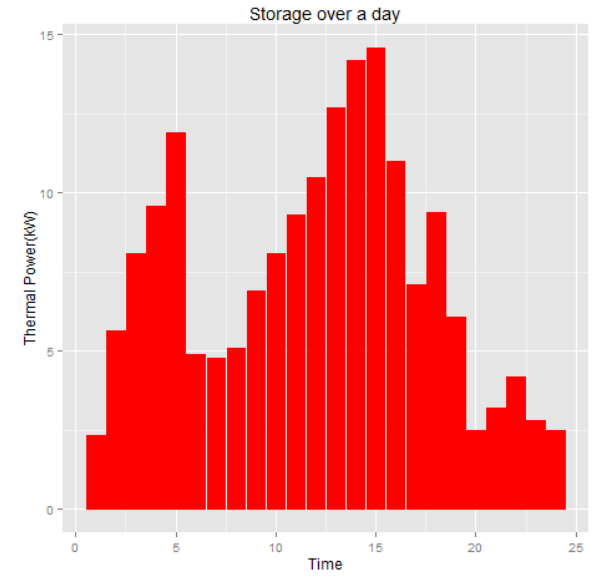

(a) Fixed Price, Summer

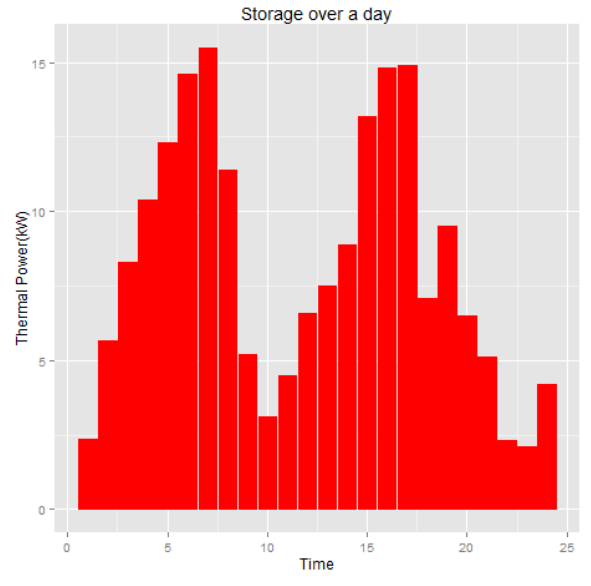

(b) Spot Prices, Summer

Figure 10: Different thermal inputs from the heat-pump and the cogeneration devices for the two cases in summer 


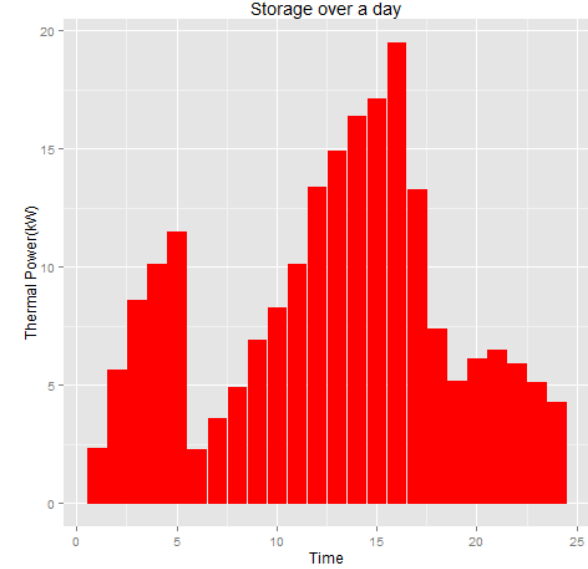

(a) Fixed Price, Winter

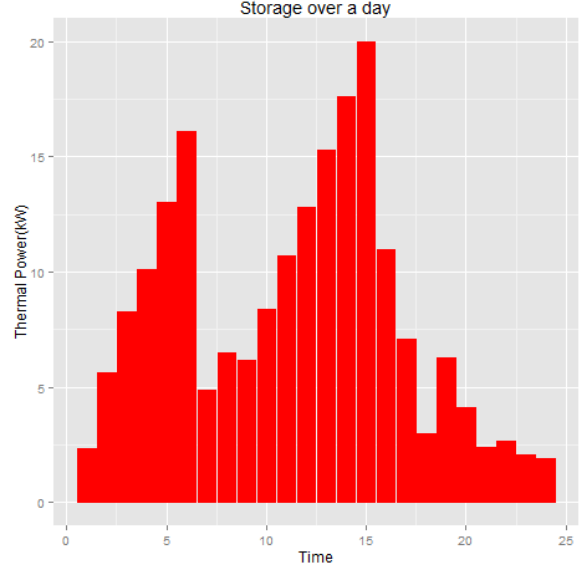

(b) Spot Prices, Winter

Figure 11: Different thermal inputs from the heat-pump and the cogeneration devices for the two cases in winter

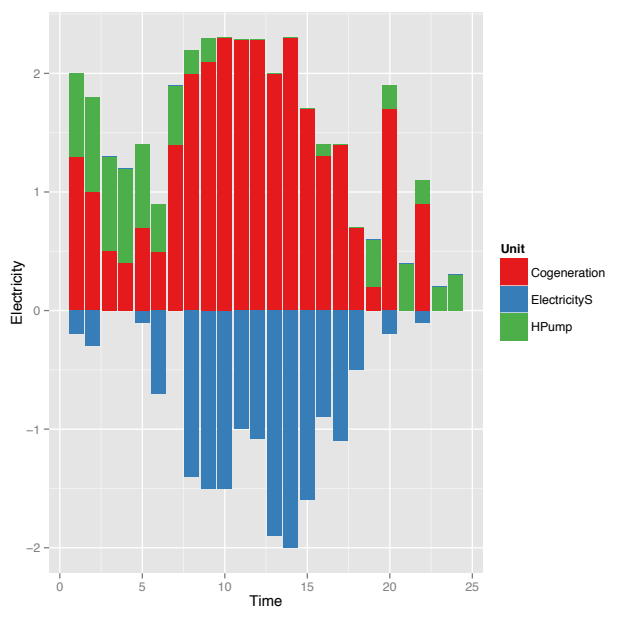

Figure 12: Strategy for 24 hours for a single family house in the microgrid for summer with fixed pricing 


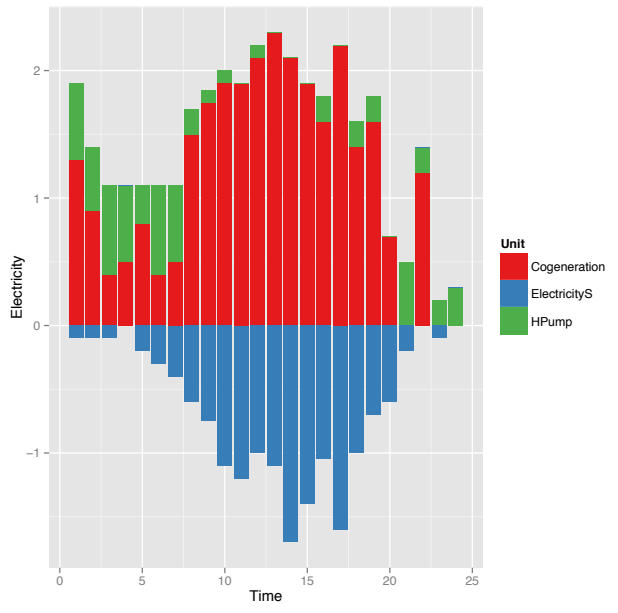

Figure 13: Strategy for 24 hours for a single family house in the microgrid for summer with fixed pricing after electrical calculations

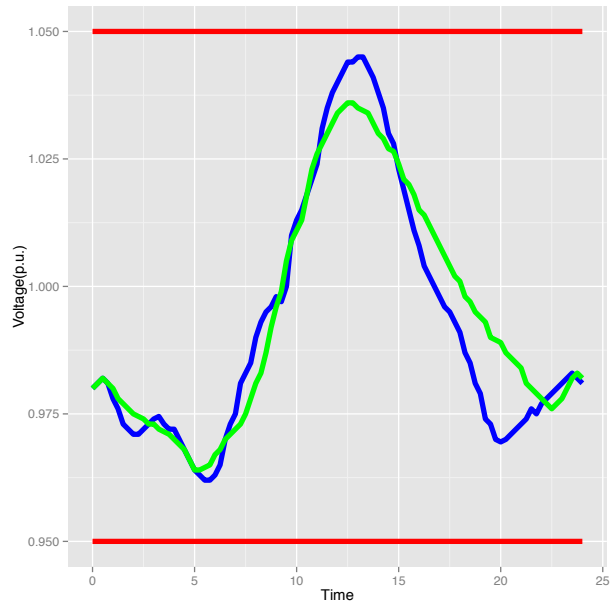

Figure 14: Voltage profile for fixed prices in summer for control with(green) and without electrical interactions(blue) 


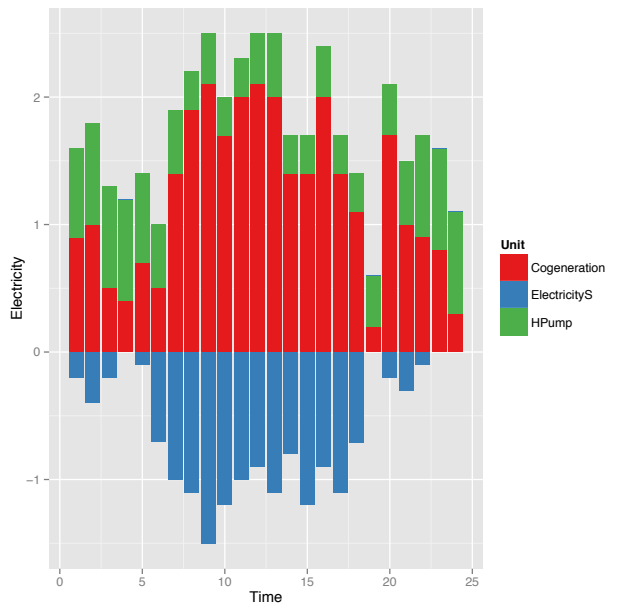

Figure 15: Strategy for 24 hours for a single family house in the microgrid for winter with fixed pricing

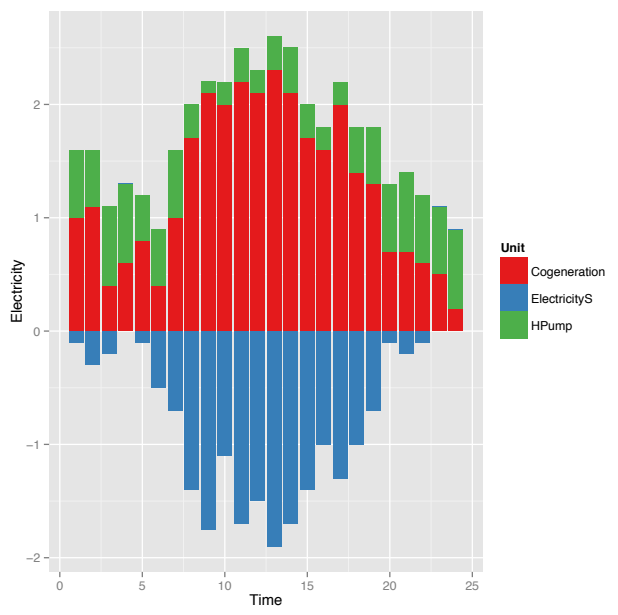

Figure 16: Strategy for 24 hours for a single family house in the microgrid for winter with fixed pricing after electrical calculations 


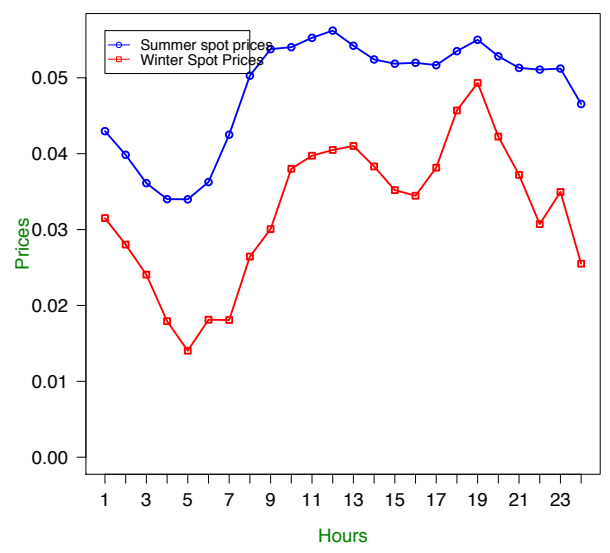

Figure 17: Spot prices from Swiss market for summer and winter for the year 2012

to sell electricity or store it, has also shifted to the right, as can be seen in Fig.18 and Fig.21. Fig.19 and Fig.22 shows the modified strategy after the introduction of the quality-of-supply problem.

Similar to Fig.14, Fig.20 shows the 24-hour voltage profile for the network for the day in summer with a direct comparison with the profile without voltage control. The difference between Fig.14 and Fig.20 is that the former is for fixed price profile while the latter is for variable prices.

\subsection{Effect of Transfer/Buffer variables}

These set of simulations also use the spot prices from the day-ahead market. Here the focus is on the difference in strategies that are brought to the front by the application and the change of the transfer variables that provides information on the ability of the system to store, or release energy in the storage devices. The same day is used to set a base case and the resulting changes in the controlled resources are shown with respect to the different strategies.

Here four additional variables $\left(\Psi, \Psi^{\prime}\right.$ and $\left.\chi, \chi^{\prime}\right)$ have been added to the existing predictive control management systems(Eq.23 and 24). The four variables 


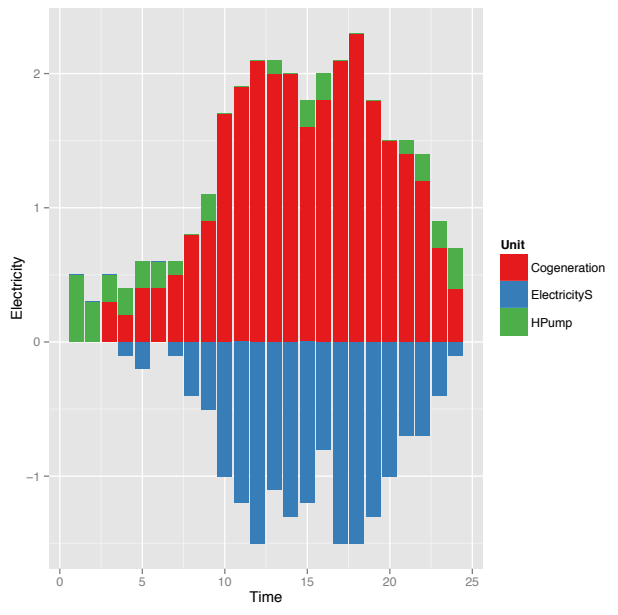

Figure 18: Strategy for 24 hours for a single family house in the microgrid for summer with variable pricing

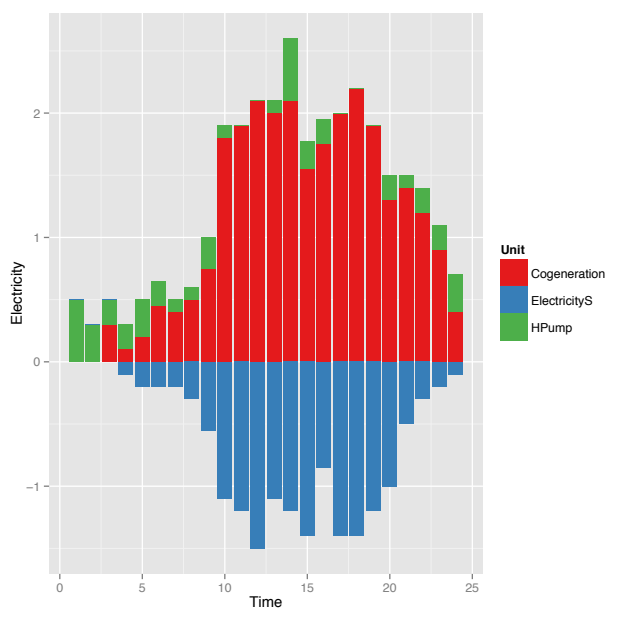

Figure 19: Strategy for 24 hours for a single family house in the microgrid for summer with variable pricing after changes in electrical calculations 


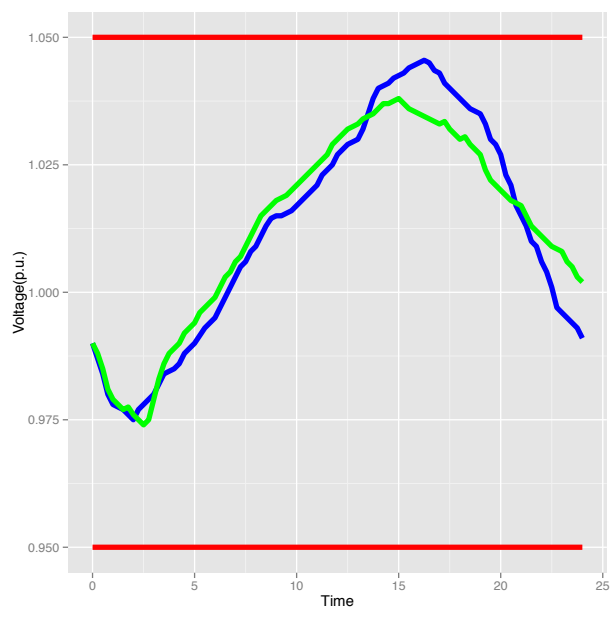

Figure 20: Voltage profile for variable prices in summer for control with(green) and without electrical interactions(blue)

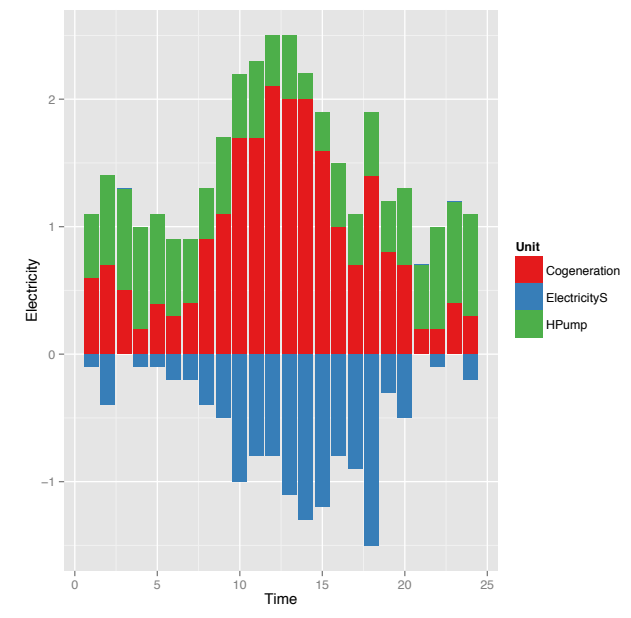

Figure 21: Strategy for 24 hours for a single family house in the microgrid for winter with variable pricing 


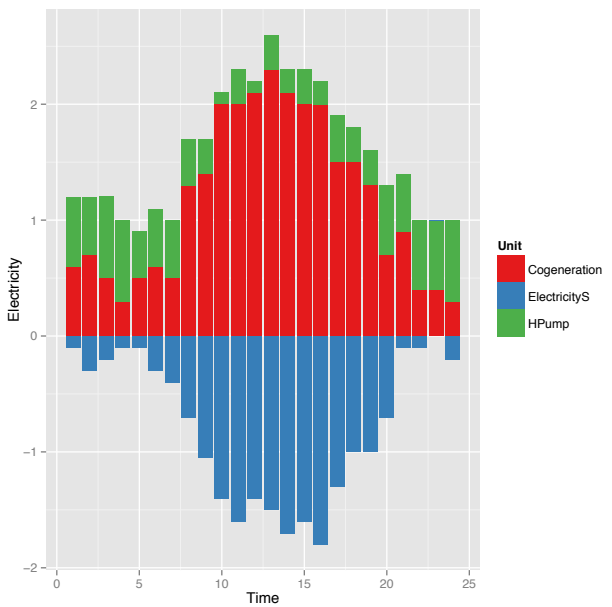

Figure 22: Strategy for 24 hours for a single family house in the microgrid for winter with variable pricing

at any given time $t$ in the network and the potential to accept from the network or provide to the network. Manipulation of the four variables result in a modification of the strategy to absorb or provide a percentage of the available energy buffer in the network by running a DEG and filling up a storage unit for the requirement of satisfaction of forecasted profiles from the utility companies or to deplete storage devices or DEGs for other networks.

This section studies the impact of the added variables which provide the MPC with the ability to see the potential of any part of the grid to absorb or provide electricity to/from the grid. This can be seen below in the Fig.23. This provides the control an advantage in the terms of providing a better strategy. This can also be used to manipulate or change the amount of electricity/heat stored at any one point.

\section{Conclusion}

The paper has proposed an intra-day model predictive control (MPC) strategy for microgrids interfacing electrical and thermal generation, storage and loads. The peculiarity of the proposed intra-day MPC is that it is able to 


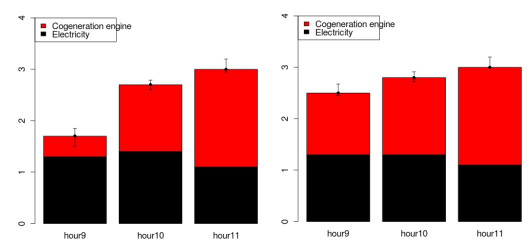

Figure 23: Difference in the strategy and the storage potentials when the variables $\left(\Psi, \Psi^{\prime}\right.$ and $\left.\chi, \chi^{\prime}\right)$ are changed from 0.8 to 0.5

bring together the electric and thermal power flow constraints under a single set of equations and a unified algorithm such that it accounts for the different inertia and, consequently, the different dynamics of the various systems and resources. The different dynamics, and inertias, are tracked using the formulation of the optimisation problem as a two-level problem. The two-level problem decomposition makes it possible to make predictions that satisfy the thermal demands of the end-users using the CHP units while minimising the objective function(operating cost, carbon emissions etc.) and simultaneously be flexible enough to keep up with the evolution of power flows in the electrical systems. This is performed by solving a secondary optimisation which treats the microgrid as a purely electrical problem. The secondary-optimisation problem is able to successfully combine the faster dynamics of the electrical systems (by maintaining the operational levels of the electrical grid within the DNOs requirements) and account for the slow dynamics of the thermal flows through the use of a feedback correction at the end of every 15 minutes. The ability to quantify the available storage buffers, together with the capability to manipulate them, is also shown and discussed through the final simulations using buffer variables. This provides the users and the utilities with the possibility of modifying and controlling the strategy creation through manipulation of the buffer variables.

The performance assessment of the proposed intra-day MPC has been carried 
out by using the microgrid test benchmark defined by the Cigre task force C6.04.02. The resulting strategy is able to satisfy the comforts of both electrical and thermal demands of the end-users while optimising the use of the available resources. At the same time, the created strategy simultaneously increases the efficiency and minimises the costs. This is realised through the shaving of peaks in demand and through the better exploitation of electrical and thermal storage.

The strategy also shows its ability to integrate stochastic resources for the satisfaction of demand. The effect of different electrical prices has been also studied. For the specific case of spot prices, the strategy created by the MPC proves its ability to create optimal patterns for thermal and electrical demands when the end-users are able to bid in the market as active members, while satisfying all constraints and comforts.

\section{References}

[1] R. H. Lasseter, Microgrids management, 2002. URL: http://ieeexplore. ieee.org/lpdocs/epic03/wrapper.htm?arnumber $=4505827$. doi:10.1109/PESW. 2002.985003.

[2] I. Beausoleil-Morrison, An experimental and simulation-based investigation of the performance of small-scale fuel cell and combustion-based cogeneration devices serving residential buildings, Technical Report April, Government of Canada, 2008. URL: http://iea-annex54.org/annex42/pdfs/ Annex_42_Final_Report.pdf.

[3] A. Collazos, F. Maréchal, C. Gähler, Computers \& Chemical Engineering 33 (2009) 1584-1592. URL: http://linkinghub.elsevier.com/ retrieve/pii/S0098135409001318. doi:10.1016/j. compchemeng. 2009. 05.009 .

[4] I. Prodan, E. Zio, International Journal of Electrical Power \& Energy Systems 61 (2014) 399-409. URL: http://www . 
sciencedirect.com/science/article/pii/S0142061514001197. doi:http://dx.doi.org/10.1016/j.ijepes.2014.03.017.

[5] P. O. Kriett, M. Salani, Energy 42 (2012) 321-330. URL: http://dx.doi . org/10.1016/j.energy.2012.03.049. doi:10.1016/j.energy.2012.03. 049.

[6] F. Katiraei, R. Iravani, N. Hatziargyriou, A. Dimeas, Power and Energy Magazine, IEEE 6 (2008) 54-65. doi:10.1109/MPE. 2008.918702.

[7] E. Barklund, N. Pogaku, M. Prodanovic, C. Hernandez-Aramburo, T. Green, in: System of Systems Engineering, 2007. SoSE '07. IEEE International Conference on, pp. 1-6. doi:10.1109/SYSOSE. 2007.4304233.

[8] A. Parisio, E. Rikos, G. Tzamalis, L. Glielmo, Applied Energy 115 (2014) 37-46. URL: http://www.sciencedirect.com/science/article/ pii/S0306261913008477. doi:10.1016/j . apenergy.2013.10.027.

[9] A. Hooshmand, H. Malki, J. Mohammadpour, Computers \& Mathematics with Applications 64 (2012) 869-876. URL: http://dx.doi .org/10.1016/ j. camwa.2012.01.028. doi:10.1016/j. camwa.2012.01.028.

[10] F. Garcia, C. Bordons, in: IECON 2013 - 39th Annual Conference of the IEEE Industrial Electronics Society, IEEE, 2013, pp. 7932-7937. URL: http://ieeexplore.ieee.org/lpdocs/epic03/ wrapper .htm?arnumber=6700458. doi:10 . 1109/IECON . 2013.6700458.

[11] F. Garcia, C. Bordons, in: IECON 2013 - 39th Annual Conference of the IEEE Industrial Electronics Society, IEEE, 2013, pp. 7962-7967. URL: http://ieeexplore.ieee.org/lpdocs/epic03/ wrapper . htm?arnumber=6700463. doi:10 . 1109/IECON . 2013 . 6700463.

[12] S. Papathanassiou, N. Hatziargyriou, K. Strunz, et al., in: Proceedings of the CIGRE Symposium: Power Systems with Dispersed Generation, pp. 1-8. 
[13] P. Stluka, D. Godbole, T. Samad, in: Decision and Control and European Control Conference (CDC-ECC), 2011 50th IEEE Conference on, pp. 51505157. doi:10.1109/CDC.2011.6161051.

[14] A. K. Molyneaux, G. B. Leyland, D Favrat, in: Proceedings of the Third International Symposium on Adaptive SystemsEvolutionary Computation and Probabilistic Graphical Models, Institute of Cybernetics, Mathematics and Physics, 2001, pp. 41-47.

[15] R. P. Menon, M. Paolone, F. Maréchal, Energy 55 (2013) 134141. URL: http://dx.doi.org/10.1016/j.energy .2013.03.070. doi:10.1016/j.energy. 2013.03.070.

[16] L. Girardin, F. Marechal, M. Dubuis, N. Calame-Darbellay, D. Favrat, Energy 35 (2010) 830-840. URL: http://linkinghub.elsevier.com/ retrieve/pii/S0360544209003582. doi:10.1016/j.energy.2009.08. 018.

${ }_{475}$ [17] K. Christakou, J. LeBoudec, M. Paolone, D.-C. Tomozei, Smart Grid, IEEE Transactions on 4 (2013) 741-750. doi:10.1109/TSG.2012.2221751.

[18] V. Dorer, A. Weber, Energy Conversion and Management 50 (2009) 648-657. URL: http://dx.doi.org/10.1016/j .enconman.2008.10.012. doi:10.1016/j.enconman.2008.10.012. 